\title{
INEQUALITIES AND MAJORISATIONS FOR THE RIEMANN-STIELTJES INTEGRAL ON TIME SCALES
}

\author{
Dorota Mozyrska, Ewa PawŁuszewicz and Delfim F. M. Torres
}

Abstract. We prove dynamic inequalities of majorisation type for functions on time scales. The results are obtained using the notion of Riemann-Stieltjes delta integral and give a generalization of [App. Math. Let., 22, 3 (2009), 416-421] to time scales.

Mathematics subject classification (2010): 26D15, 26E70, 39A12.

Keywords and phrases: Time scales, Riemann-Stieltjes delta integrals, dynamic inequalities, inequalities of majorisation type.

\section{REFERENCES}

[1] R. Agarwal, M. Bohner And A. Peterson, Inequalities on time scales: a survey, Math. Inequal. Appl., 4, 4 (2001), 535-557.

[2] B. Aulbach and L. Neidhart, Integration on measure chains, Proceedings of the Sixth International Conference on Difference Equations, 239-252, CRC, Boca Raton, FL, 2004.

[3] N. S. BARnett, P. CERone ANd S. S. Dragomir, Majorisation inequalities for Stieltjes integrals, App. Math. Let., 22, 3 (2009), 416-421.

[4] M. Bohner And G. Sh. Guseinov, Multiple integration on time scales, Dynam. Systems Appl., 14, 3-4 (2005), 579-606.

[5] M. Bohner And G. Sh. Guseinov, Multiple Lebesgue integration on time scales, Adv. Difference Equ. 2006, Art. ID 26391, 12 pp.

[6] M. Bohner And G. SH. Guseinov, Double integral calculus of variations on time scales, Comput. Math. Appl., 54, 1 (2007), 45-57.

[7] M. Bohner And A. Peterson, Advances in dynamic equations on time scales, Birkhäuser Boston, Boston, MA, 2003.

[8] M. Carter And B. van Brunt, The Lebesgue-Stieltjes integral, Springer, New York, 2000.

[9] S. S. DRAGOMIR, Inequalities for Stieltjes integrals with convex integrators and applications, Appl. Math. Lett., 20, 2 (2007), 123-130.

[10] R. A. C. Ferreira AND D. F. M. Torres, Some linear and nonlinear integral inequalities on time scales in two independent variables, Nonlinear Dyn. Syst. Theory, 9, 2 (2009), 161-169.

[11] R. A. C. FERreira AND D. F. M. TORRES, Generalizations of Gronwall-Bihari inequalities on time scales, J. Difference Equ. Appl., 15, 6 (2009), 529-539.

[12] G. Sh. Guseinov, Integration on time scales, J. Math. Anal. Appl., 285, 1 (2003), 107-127.

[13] D. Mozyrska, E. PawŁuszewicz and D. F. M. Torres, The Riemann-Stieltjes integral on time scales, Aust. J. Math. Anal. Appl., 7, 1 (2010), Art. 10, 14 pp.

[14] S. SAILER, Riemann-Stieltjes Integrale auf Zeitmengen (Schriftliche Hausarbeit, vorgelegt bei Prof. Dr. B. Aulbach), Universität Augsburg, 1992.

[15] M. R. Sidi Ammi, R. A. C. Ferreira AND D. F. M. Torres, Diamond- $\alpha$ Jensen's inequality on time scales, J. Inequal. Appl. 2008, Art. ID 576876, 13 pp.

[16] M. R. Sidi Ammi And D. F. M. Torres, Combined dynamic Grüss inequalities on time scales, J. Math. Sci. (N. Y.), 161, 6 (2009), 792-802.

[17] C.-C. Yen, F.-H. Wong AND H.-J. LI, Čebyšev's inequality on time scales, JIPAM. J. Inequal. Pure Appl. Math., 6, 1 (2005), Article 7, 10 pp. 